\title{
Photons, neutrinos, and optical activity
}

\author{
Ali Abbasabadi ${ }^{1}$ and Wayne W. Repko ${ }^{2}$ \\ ${ }^{1}$ Department of Physical Sciences, Ferris State University, Big Rapids, Michigan 49307, USA \\ ${ }^{2}$ Department of Physics and Astronomy, Michigan State University, East Lansing, Michigan 48824, USA
}

(Dated: November 2, 2018)

\begin{abstract}
We compute the one-loop helicity amplitudes for low-energy $\nu \gamma \rightarrow \nu \gamma$ scattering and its crossed channels in the standard model with massless neutrinos. In the center of mass, with $\sqrt{s}=2 \omega \ll 2 m_{e}$, the cross sections for these $2 \rightarrow 2$ channels grow roughly as $\omega^{6}$. The scattered photons in the elastic channel are circularly polarized and the net value of the polarization is non-zero. We also present a discussion of the optical activity of a sea of neutrinos and estimate the values of its index of refraction and rotary power.
\end{abstract}

PACS numbers: 13.15.+g, 14.60.Lm, 14.70.Bh, 95.30.Cq

\section{INTRODUCTION}

Neutrino-photon elastic scattering $\nu \gamma \rightarrow \nu \gamma$ and its crossed channels, which are of interest in astrophysical applications, have been studied using a variety of models for the weak interaction [1, 2, 3, 田, 5, 6]. When the center of mass energy $\sqrt{s}$ is much less than twice the electron mass $2 m_{e}$, the amplitudes for these processes with massless neutrinos are of leading order $s^{2} / m_{W}^{4}$, where $m_{W}$ is $W$-boson mass [5]. This dependence leads to low-energy cross sections which grow as $s^{3}$, with a scale set by $m_{W}$. Apart from a factor $\ln \left(m_{W}^{2} / m_{e}^{2}\right)$, there is no dependence on $m_{e}$ in leading order. Here, we present expressions for the low-energy neutrino-photon helicity amplitudes which are valid to higher orders in $s$, and contain terms whose scale is set by $m_{e}^{2}$. We find that the helicity flip amplitudes for the $\nu \gamma \rightarrow \nu \gamma$ and the helicity non-flip amplitudes for the $\gamma \gamma \rightarrow \nu \bar{\nu}$, which vanish in leading order, are non-zero. Furthermore, the inclusion of higher powers of $s$, such as $s^{3} / m_{W}^{6}$, enables us to use the forward elastic scattering amplitudes for $\nu \gamma \rightarrow \nu \gamma$ to study the optical activity of a sea of neutrinos [7].

In the next section, we use invariant decompositions of the amplitudes for $\nu \gamma \rightarrow \nu \gamma$ and its crossed channels to obtain properties of and restrictions on the corresponding helicity amplitudes. Section III gives the numerical results for the complete one-loop helicity dependent differential and total cross sections. This is followed by a discussion and conclusions, which include a treatment of the production of circularly polarized photons in low energy $\nu \gamma \rightarrow \nu \gamma$ scattering. We also calculate the index of refraction and the rotary power for a sea of neutrinos as a function of the energy of an incident photon and the temperature of the neutrinos.

\section{HELICITY AMPLITUDES}

The general expressions for the Lorentz-invariant, Bose symmetric, and time reversal invariant helicity amplitudes, $\mathcal{A}_{\lambda_{1} \lambda_{2}}^{\nu \gamma \rightarrow \nu \gamma}(s, t, u)$, for the process $\nu \gamma \rightarrow \nu \gamma$ can be found in Refs. [8, 9 . They are

$$
\begin{aligned}
& \mathcal{A}_{++}^{\nu \gamma \rightarrow \nu \gamma}(s, t, u)=s u \cos (\theta / 2) \mathcal{F}(s, t, u) \\
& \mathcal{A}_{--}^{\nu \gamma \rightarrow \nu \gamma}(s, t, u)=-s^{2} \cos (\theta / 2) \mathcal{F}(u, t, s) \\
& \mathcal{A}_{+-}^{\nu \gamma \rightarrow \nu \gamma}(s, t, u)=s t \cos (\theta / 2)[\mathcal{G}(s, t, u)-\mathcal{G}(u, t, s)], \\
& \mathcal{A}_{-+}^{\nu \gamma \rightarrow \nu \gamma}(s, t, u)=\mathcal{A}_{+-}^{\nu \gamma \rightarrow \nu \gamma}(s, t, u)
\end{aligned}
$$

where the Mandelstam variables $s, t$, and $u$ are defined by $s=\left(p_{1}+k_{1}\right)^{2}, t=\left(p_{1}-p_{2}\right)^{2}=-\frac{1}{2} s(1-z)$, $u=\left(p_{1}-k_{2}\right)^{2}=-\frac{1}{2} s(1+z)$, and $z=\cos \theta$, with $\theta$ the angle between the incoming neutrino, which is moving in the $+z$ direction, and the outgoing neutrino. The helicity of the incoming photon is $\lambda_{1}= \pm 1$ and the helicity of the outgoing photon is $\lambda_{2}= \pm 1$. Here, the 4-momenta of the incoming neutrino and photon are $p_{1}$ and $k_{1}$, respectively, with $p_{2}$ and $k_{2}$ denoting the corresponding outgoing momenta. To ensure the conservation for the angular momentum in Eqs. (11)-(3), it is necessary to require that the functions $\mathcal{F}(s, t, u), \mathcal{F}(u, t, s)$, and $[\mathcal{G}(s, t, u)-\mathcal{G}(u, t, s)]$ be non-singular in the limit $u \rightarrow 0$ (backward scattering). In addition, the function $[\mathcal{G}(s, t, u)-\mathcal{G}(u, t, s)]$ must also be non-singular in the limit $t \rightarrow 0$ (forward scattering).

From the Eqs. (1) - (4), the interchange of $s$ and $u$ results in the following relation

$$
\mathcal{A}_{\lambda_{1} \lambda_{2}}^{\nu \gamma \rightarrow \nu \gamma}(s, t, u)=\mathcal{A}_{-\lambda_{1}-\lambda_{2}}^{\nu \gamma \rightarrow \nu \gamma}(u, t, s)
$$

where, under this interchange, we have changed the factor $s \cos (\theta / 2)=s \sqrt{-u / s}$ to $u \sqrt{-s / u}=-s \sqrt{-u / s}=$ $-s \cos (\theta / 2)$.

A similar decomposition of the helicity amplitudes $\mathcal{A}_{\lambda_{1} \lambda_{2}}^{\gamma \gamma \rightarrow \nu \bar{\nu}}(s, t, u)$ for the crossed channel process $\gamma \gamma \rightarrow \nu \bar{\nu}$, 
expressions are

$$
\begin{aligned}
& \mathcal{A}_{-+}^{\gamma \gamma \rightarrow \nu \bar{\nu}}(s, t, u)=\frac{1}{2} s u \sin \theta \mathcal{F}(t, s, u), \\
& \mathcal{A}_{+-}^{\gamma \gamma \rightarrow \nu \bar{\nu}}(s, t, u)=-\frac{1}{2} s t \sin \theta \mathcal{F}(u, s, t), \\
& \mathcal{A}_{--}^{\gamma \gamma \rightarrow \nu \bar{\nu}}(s, t, u)=\frac{1}{2} s^{2} \sin \theta[\mathcal{G}(t, s, u)-\mathcal{G}(u, s, t)], \\
& \mathcal{A}_{++}^{\gamma \gamma \rightarrow \nu \bar{\nu}}(s, t, u)=\mathcal{A}_{--}^{\gamma \gamma \rightarrow \nu \bar{\nu}}(s, t, u),
\end{aligned}
$$

with $s, t$, and $u$ defined by $s=\left(k_{1}+k_{2}\right)^{2}, t=\left(k_{1}-p_{1}\right)^{2}=$ $-\frac{1}{2} s(1-z), u=\left(k_{1}-p_{2}\right)^{2}=-\frac{1}{2} s(1+z)$, and $z=$ $\cos \theta$, where $\theta$ is the angle between the incoming photon 1 , which is moving in the $+z$ direction, and the outgoing neutrino. The helicities of the incoming photons are $\lambda_{1}=$ \pm 1 and $\lambda_{2}= \pm 1$, and the incoming photons have 4momenta $k_{1}$ and $k_{2}$, while $p_{1}$ and $p_{2}$ are the momenta of the outgoing neutrino and anti-neutrino, respectively. In this case, conservation of angular momentum in Eqs. (6) (8) requires that the functions $\mathcal{F}(t, s, u), \mathcal{F}(u, s, t)$, and $[\mathcal{G}(t, s, u)-\mathcal{G}(u, s, t)]$ be non-singular in the limit $u \rightarrow 0$. This is sufficient to make these functions non-singular in the limit $t \rightarrow 0$.

Using Eqs. (6) -(9), the interchange of $t$ and $u$ results in the following relation

$$
\mathcal{A}_{\lambda_{1} \lambda_{2}}^{\gamma \gamma \rightarrow \bar{\nu}}(s, t, u)=-\mathcal{A}_{-\lambda_{1}-\lambda_{2}}^{\gamma \gamma \rightarrow \nu \bar{\nu}}(s, u, t) .
$$

In addition, the comparison of Eqs. (11) - (4) with Eqs. (6) (9) shows that

$$
\mathcal{A}_{\lambda_{1} \lambda_{2}}^{\gamma \gamma \rightarrow \bar{\nu}}(t, s, u)=-i \mathcal{A}_{-\lambda_{1} \lambda_{2}}^{\nu \gamma \rightarrow \nu \gamma}(s, t, u) .
$$

Here, under the interchange of $s$ and $t$, we have changed the factor $s \sin \theta=2 s \sqrt{t u / s^{2}}$ to $2 t \sqrt{s u / t^{2}}=-2 \sqrt{s u}=$ $-2 i s \sqrt{(1+z) / 2}=-2 i s \cos (\theta / 2)$.

Using the invariance of the helicity amplitudes under the CPT operation, we obtain the helicity amplitudes for the processes $\bar{\nu} \gamma \rightarrow \bar{\nu} \gamma$ and $\nu \bar{\nu} \rightarrow \gamma \gamma$ from those of $\nu \gamma \rightarrow \nu \gamma$ and $\gamma \gamma \rightarrow \nu \bar{\nu}$, respectively. The results are

$$
\begin{aligned}
& \mathcal{A}_{\lambda_{1} \lambda_{2}}^{\bar{\nu} \gamma \rightarrow \bar{\nu} \gamma}(s, t, u)=\mathcal{A}_{-\lambda_{1}-\lambda_{2}}^{\nu \gamma \rightarrow \nu \gamma}(s, t, u), \\
& \mathcal{A}_{\lambda_{1} \lambda_{2}}^{\bar{\nu} \rightarrow \gamma \gamma}(s, t, u)=\mathcal{A}_{-\lambda_{1}-\lambda_{2}}^{\gamma \gamma \rightarrow \nu \bar{\nu}}(s, t, u) .
\end{aligned}
$$

We have calculated the amplitudes for the diagrams of Fig. 1, for $\nu \gamma \rightarrow \nu \gamma$ and its crossed channels, in a nonlinear $R_{\xi}$ gauge such that the coupling between the photon, the $W$-boson, and the Goldstone boson $(\phi)$ vanishes 15, 11, 12. Since the Goldstone boson-electron couplings introduce a factor $m_{e}^{2} / m_{W}^{2}$, and we are keeping terms of this order in our amplitudes, the contribution from the Goldstone boson, in the diagrams of Fig. 1. must be included. For zero neutrino mass, the two sets of $W$ exchange and $\phi$-exchange diagrams are separately gauge invariant. Also, the contributions of these two sets of diagrams to the helicity amplitudes for $\nu \gamma \rightarrow \nu \gamma$ separately have the structure of Eqs. (1)-(田). This is also true for
Using the algebraic manipulation software FORM 13 and SCHOONSCHIP [14], we have expressed the diagrams in terms of Feynman parameter integrals, and for $\sqrt{s} \ll$ $2 m_{e}$, have expanded these amplitudes in a power series in $s / m_{e}^{2}, t / m_{e}^{2}$, and $m_{e}^{2} / m_{W}^{2}$. The results of the calculation for the functions $\mathcal{F}(s, t, u)$ and $\mathcal{G}(s, t, u)-\mathcal{G}(u, t, s)$, are

$$
\begin{aligned}
\mathcal{F}(s, t, u) & =\frac{\alpha^{2}}{8 m_{W}^{4} \sin ^{2} \theta_{W}} f(s, t, u), \\
\mathcal{G}(s, t, u)-\mathcal{G}(u, t, s) & =\frac{\alpha^{2}}{8 m_{W}^{4} \sin ^{2} \theta_{W}} g(s, t, u),
\end{aligned}
$$

where

$$
\begin{aligned}
f(s, t, u)= & -4-\frac{16}{3} \ln \left(\frac{m_{W}^{2}}{m_{e}^{2}}\right)-\frac{22}{45} \frac{t}{m_{e}^{2}} \\
& -\frac{11}{315} \frac{t^{2}}{m_{e}^{4}}-\frac{37}{9450} \frac{t^{3}}{m_{e}^{6}}-\frac{4}{7425} \frac{t^{4}}{m_{e}^{8}} \\
& +\frac{m_{e}^{2}}{m_{W}^{2}}\left[\left(\frac{8}{3}-\frac{8}{3} \frac{s}{m_{e}^{2}}+\frac{8}{3} \frac{t}{m_{e}^{2}}\right) \ln \left(\frac{m_{W}^{2}}{m_{e}^{2}}\right)\right. \\
& -10+\frac{64}{9} \frac{s}{m_{e}^{2}}-\frac{163}{45} \frac{t}{m_{e}^{2}}-\frac{4}{45} \frac{s t}{m_{e}^{4}} \\
& +\frac{169}{630} \frac{t^{2}}{m_{e}^{4}}-\frac{1}{315} \frac{s t^{2}}{m_{e}^{6}}+\frac{71}{3780} \frac{t^{3}}{m_{e}^{6}} \\
& \left.-\frac{1}{4725} \frac{s t^{3}}{m_{e}^{8}}+\frac{106}{51975} \frac{t^{4}}{m_{e}^{8}}\right], \\
g(s, t, u)= & \frac{s-u}{m_{e}^{2}}\left[\frac{2}{45}+\frac{1}{315} \frac{t}{m_{e}^{2}}+\frac{1}{3150} \frac{t^{2}}{m_{e}^{4}}\right. \\
& +\frac{2}{51975} \frac{t^{3}}{m_{e}^{6}}+\frac{m_{e}^{2}}{m_{W}^{2}}\left(-\frac{17}{45}-\frac{23}{630} \frac{t}{m_{e}^{2}}\right. \\
& \left.\left.-\frac{1}{420} \frac{t^{2}}{m_{e}^{4}}-\frac{23}{103950} \frac{t^{3}}{m_{e}^{6}}\right)\right] .
\end{aligned}
$$

Here, $\theta_{W}$ is the weak mixing angle, $\alpha$ is the fine structure constant, and we have neglected higher powers of $s / m_{e}^{2}$, $t / m_{e}^{2}$, and $m_{e}^{2} / m_{W}^{2}$. The first two terms of $f(s, t, u)$ in Eq. (16) were previously derived in the Ref. [5]. These results show that there are many higher order terms whose scale is set by $m_{e}^{2}$. Note, however, that in the forward direction $(t=0)$ the scale in Eq. (16) is set by $m_{W}^{2}$. This suggests that Eq. (16) is valid even for $\sqrt{s}>m_{e}$ when $t=0$.

We can confirm the validity of the forward limit of Eq. (16) for the range $\sqrt{s} \ll m_{W}$ by using the dispersion relation

$$
\begin{aligned}
\mathcal{A}_{\lambda \lambda}^{\nu \gamma \rightarrow \nu \gamma}(s, 0,-s)= & \frac{s^{2}}{\pi} \int_{\left(m_{W}+m_{e}\right)^{2}}^{\infty} \frac{d s^{\prime}}{s^{\prime}} \\
& \times\left(\frac{\sigma_{\lambda}\left(s^{\prime}\right)}{s^{\prime}-s}+\frac{\sigma_{\lambda}\left(-s^{\prime}\right)}{s^{\prime}+s}\right),
\end{aligned}
$$

to obtain the exact value of the non-flip forward helicity amplitude $\mathcal{A}_{\lambda \lambda}^{\nu \gamma \rightarrow \nu \gamma}(s, 0,-s)$ for $s<\left(m_{W}+m_{e}\right)^{2}$. The 
for the process $\nu \gamma \rightarrow e^{-} W^{+}$, after summation over the helicities of the $W$-boson and the electron ( $\lambda$ is helicity of the photon). Using Eqs. (5) and (18), it can be shown that the following symmetry relation must exist

$$
\sigma_{\lambda}\left(-s^{\prime}\right)=\sigma_{-\lambda}\left(s^{\prime}\right) .
$$

Therefore, we can write [8]

$$
\begin{aligned}
\mathcal{A}_{\lambda \lambda}^{\nu \gamma \rightarrow \nu \gamma}(s, 0,-s)= & \frac{s^{2}}{\pi} \int_{\left(m_{W}+m_{e}\right)^{2}}^{\infty} \frac{d s^{\prime}}{s^{\prime}} \\
& \times\left(\frac{\sigma_{\lambda}\left(s^{\prime}\right)}{s^{\prime}-s}+\frac{\sigma_{-\lambda}\left(s^{\prime}\right)}{s^{\prime}+s}\right) .
\end{aligned}
$$

A calculation of $\sigma_{\lambda}(s)$ gives

$$
\begin{aligned}
\sigma_{\lambda}(s)= & \sqrt{2} G_{F} \alpha\left[\left(\frac{2}{s}-\lambda \frac{m_{e}^{2}}{s^{2}}+6 \lambda \frac{m_{W}^{2}}{s^{2}}-2 \frac{m_{e}^{2} m_{W}^{2}}{s^{3}}\right.\right. \\
& \left.-2 \frac{m_{e}^{4}}{s^{3}}+4 \frac{m_{W}^{4}}{s^{3}}\right) \sqrt{\mu\left(s, m_{e}^{2}, m_{W}^{2}\right)} \\
& +\left(-2 \lambda \frac{m_{W}^{2}}{s}+\frac{m_{e}^{2} m_{W}^{2}}{s^{2}}+3 \lambda \frac{m_{e}^{2} m_{W}^{2}}{s^{2}}+\frac{m_{e}^{4}}{s^{2}}\right. \\
& -2 \frac{m_{W}^{4}}{s^{2}}+2 \lambda \frac{m_{W}^{4}}{s^{2}}+\frac{m_{e}^{2} m_{W}^{4}}{s^{3}}-2 \frac{m_{e}^{4} m_{W}^{2}}{s^{3}} \\
& \left.-\frac{m_{e}^{6}}{s^{3}}+2 \frac{m_{W}^{6}}{s^{3}}\right) \ell\left(s, m_{e}^{2}, m_{W}^{2}\right) \\
& +\left(\frac{1}{2} \frac{m_{e}^{2}}{s}+\frac{\lambda}{2} \frac{m_{e}^{2}}{s}+\frac{m_{W}^{2}}{s}-\lambda \frac{m_{W}^{2}}{s}\right. \\
& +\frac{m_{e}^{2} m_{W}^{2}}{s^{2}}+3 \lambda \frac{m_{e}^{2} m_{W}^{2}}{s^{2}}-2 \frac{m_{W}^{4}}{s^{2}}+2 \lambda \frac{m_{W}^{4}}{s^{2}} \\
& +\frac{m_{e}^{4}}{s^{2}}+\frac{m_{e}^{2} m_{W}^{4}}{s^{3}}-2 \frac{m_{e}^{4} m_{W}^{2}}{s^{3}} \\
& \left.\left.-\frac{m_{e}^{6}}{s^{3}}+2 \frac{m_{W}^{6}}{s^{3}}\right) \ell\left(s, m_{W}^{2}, m_{e}^{2}\right)\right],
\end{aligned}
$$

where

$$
\begin{aligned}
& \mu(x, y, z)=x^{2}+y^{2}+z^{2}-2 x y-2 x z-2 y z \\
& \ell(x, y, z)=\ln \left(\frac{x-y+z+\sqrt{\mu(x, y, z})}{x-y+z-\sqrt{\mu(x, y, z})}\right)
\end{aligned}
$$

and $G_{F}=\pi \alpha /\left(\sqrt{2} m_{W}^{2} \sin ^{2} \theta_{W}\right)$ is the Fermi coupling. After setting the electron mass to zero everywhere but in the logarithm, our spin averaged cross section $\left(\sigma_{+}+\right.$ $\left.\sigma_{-}\right) / 2$ agrees with the result previously obtained by Seckel [15].

Assuming $\sqrt{s} \ll m_{W}$, an expansion of the dispersion integral Eq. (20) to order $s^{3} / m_{W}^{6}$ gives

$$
\begin{aligned}
& \mathcal{A}_{++}^{\nu \gamma \rightarrow \nu \gamma}(s, 0,-s)=\frac{-\alpha^{2} s^{2}}{8 m_{W}^{4} \sin ^{2} \theta_{W}} f(s, 0,-s), \\
& \mathcal{A}_{--}^{\nu \gamma \rightarrow \nu \gamma}(s, 0,-s)=\frac{-\alpha^{2} s^{2}}{8 m^{4} \sin ^{2} \theta_{I X}} f(-s, 0, s),
\end{aligned}
$$

where

$$
\begin{aligned}
f(s, 0,-s)= & -4-\frac{16}{3} \ln \left(\frac{m_{W}^{2}}{m_{e}^{2}}\right) \\
& +\frac{m_{e}^{2}}{m_{W}^{2}}\left[\left(\frac{8}{3}-\frac{8}{3} \frac{s}{m_{e}^{2}}\right) \ln \left(\frac{m_{W}^{2}}{m_{e}^{2}}\right)\right. \\
& \left.-10+\frac{64}{9} \frac{s}{m_{e}^{2}}\right],
\end{aligned}
$$

and we have neglected terms in higher powers of $m_{e}^{2} / m_{W}^{2}$. The function $f(-s, 0, s)$ can be found from Eq. (26) by replacing $s$ by $-s$. Notice that Eqs. (24)-(26) are consistent with Eqs. (5) and (16), and although Eq. (16) was derived for $\sqrt{s} \ll 2 m_{e}$, its range of validity for $t=0$, as suggested above, extends to $\sqrt{s} \ll m_{W}$.

\section{DIFFERENTIAL AND TOTAL CROSS SECTIONS}

In Fig.2, we show the differential cross sections, for $\nu \gamma \rightarrow \nu \gamma$, using

$$
\frac{d \sigma_{\lambda_{1} \lambda_{2}}^{\nu \gamma \rightarrow \nu \gamma}}{d z}=\frac{1}{32 \pi s}\left|\mathcal{A}_{\lambda_{1} \lambda_{2}}^{\nu \gamma \rightarrow \nu \gamma}\right|^{2},
$$

where $\lambda_{1}$ and $\lambda_{2}$ are the helicities of the incoming and outgoing photons, respectively. Here, $z=\cos \theta$, and $\theta$ is the angle between the incoming neutrino, which is moving in the $+z$ direction, and the outgoing neutrino. Figure 2 shows the identity of the two helicity amplitudes $\mathcal{A}_{+-}^{\nu \gamma \rightarrow \nu \gamma}$ and $\mathcal{A}_{-+}^{\nu \gamma \rightarrow \nu \gamma}$ given by Eq. (价. It also shows the vanishing of the amplitudes for backward scattering, and vanishing of the flip amplitudes for forward scattering, as given in Eqs. (11)-(1).

The total cross sections for helicities $\lambda_{1}$ and $\lambda_{2}$ are given by

$$
\sigma_{\lambda_{1} \lambda_{2}}^{\nu \gamma \rightarrow \nu \gamma}=\int_{-1}^{1} \frac{d \sigma_{\lambda_{1} \lambda_{2}}^{\nu \gamma \rightarrow \nu \gamma}}{d z} d z
$$

and are plotted in Fig. 3. Also shown in dots is the helicity flip cross section, which can be seen to be much smaller than the cross sections for helicity non-flip. This feature seems not to be a consequence of any symmetry. Figure 3 illustrates the roughly $s^{3}$ behavior of the total cross section for helicity non-flip, and $s^{5}$ behavior for the helicity flip, at photon energies $\omega \ll m_{e}$. A fit to the points in Fig. 3 gives

$$
\begin{aligned}
& \sigma_{--}^{\nu \gamma \rightarrow \nu \gamma}=3.9 \times 10^{-32}\left(\frac{\omega}{m_{e}}\right)^{6} \mathrm{pb}, \\
& \sigma_{++}^{\nu \gamma \rightarrow \nu \gamma}=2.0 \times 10^{-32}\left(\frac{\omega}{m_{e}}\right)^{6} \mathrm{pb}, \\
& \sigma_{-+}^{\nu \gamma \rightarrow \nu \gamma}=2.2 \times 10^{-38}\left(\frac{\omega}{m}\right)^{10} \mathrm{pb},
\end{aligned}
$$


with

$$
\sigma_{+-}^{\nu \gamma \rightarrow \nu \gamma}=\sigma_{-+}^{\nu \gamma \rightarrow \nu \gamma} .
$$

Here, $\omega=\sqrt{s} / 2$ is the energy of a photon (or a neutrino), and $m_{e}$ is the mass of the electron. Therefore, the total cross section for an unpolarized initial photon can be approximated as

$$
\sigma^{\nu \gamma \rightarrow \nu \gamma}=3.0 \times 10^{-32}\left(\frac{\omega}{m_{e}}\right)^{6} \mathrm{pb}, \quad \omega \ll m_{e} .
$$

This is the same $\omega^{6}$ behavior as found in the numerical calculations of Ref. [8] for the region $m_{e} \ll \omega \ll m_{W}$, but with a larger slope.

In Fig. 1, we show the differential cross sections for $\gamma \gamma \rightarrow \nu \bar{\nu}$, using

$$
\frac{d \sigma_{\lambda_{1} \lambda_{2}}^{\gamma \gamma \rightarrow \nu \bar{\nu}}}{d z}=\frac{1}{32 \pi s}\left|\mathcal{A}_{\lambda_{1} \lambda_{2}}^{\gamma \gamma \rightarrow \nu \bar{\nu}}\right|^{2},
$$

where $\lambda_{1}$ and $\lambda_{2}$ are the helicities of the incoming photons. In this case, $z=\cos \theta$, with $\theta$ being the angle between the incoming photon 1 , which is moving in the $+z$ direction, and the outgoing neutrino. The identity of the two helicity amplitudes $\mathcal{A}_{++}^{\gamma \gamma \rightarrow \nu \bar{\nu}}$ and $\mathcal{A}_{--}^{\gamma \gamma \rightarrow \nu \bar{\nu}}$, as implied by Eq. (9), is also shown in this figure, as is the vanishing of the amplitudes for the forward and backward scattering, implied by Eqs. (6)-(9). Notice also that this figure clearly exhibits the symmetry relations of Eq. (10).

The total cross sections for $\gamma \gamma \rightarrow \nu \bar{\nu}$ are plotted in Fig. 5, using

$$
\sigma_{\lambda_{1} \lambda_{2}}^{\gamma \gamma \rightarrow \nu \bar{\nu}}=\int_{-1}^{1} \frac{d \sigma_{\lambda_{1} \lambda_{2}}^{\gamma \gamma \rightarrow \nu \bar{\nu}}}{d z} d z
$$

Shown in dots is the cross section for the helicity non-flip, which can be seen to be much smaller than the helicity flip cross sections. Again, we see a roughly $s^{3}$ behavior of the dominant contributions to the total cross section, and a $s^{5}$ behavior of the non-leading contributions, at photon energies $\omega \ll m_{e}$. Fits to the points in this case give

$$
\begin{aligned}
& \sigma_{--}^{\gamma \gamma \rightarrow \nu \bar{\nu}}=4.0 \times 10^{-33}\left(\frac{\omega}{m_{e}}\right)^{6} \mathrm{pb}, \\
& \sigma_{-+}^{\gamma \gamma \rightarrow \nu \bar{\nu}}=5.5 \times 10^{-39}\left(\frac{\omega}{m_{e}}\right)^{10} \mathrm{pb},
\end{aligned}
$$

with

$$
\begin{aligned}
& \sigma_{++}^{\gamma \gamma \rightarrow \nu \bar{\nu}}=\sigma_{--}^{\gamma \gamma \rightarrow \nu \bar{\nu}}, \\
& \sigma_{+-}^{\gamma \gamma \rightarrow \nu \bar{\nu}}=\sigma_{-+}^{\gamma \gamma \rightarrow \nu \bar{\nu}},
\end{aligned}
$$

which are the consequences of Eq. (10). The total cross section for the unpolarized initial photons can be approximated as

$$
\sigma^{\gamma \gamma \rightarrow \nu \bar{\nu}}=2.0 \times 10^{-33}\left(\frac{\omega}{m}\right)^{6} \mathrm{pb}, \quad \omega \ll m_{e} .
$$

In this case, too, the $\omega^{6}$ dependence is the same as that found in Ref. 10] for $m_{e} \ll \omega \ll m_{W}$, but with a large slope.

The differential cross sections for the process $\bar{\nu} \nu \rightarrow \gamma \gamma$, for photons with helicities $\lambda_{1}$ and $\lambda_{2}$, can be obtained from the following relation

$$
\frac{d \sigma_{\lambda_{1} \lambda_{2}}^{\bar{\nu} \nu \rightarrow \gamma \gamma}}{d z}=\frac{d \sigma_{-\lambda_{1}-\lambda_{2}}^{\gamma \gamma \rightarrow \nu \bar{\nu}}}{d z}
$$

where $z=\cos \theta$, and on the left side of this equation, $\theta$ is the angle between the incoming anti-neutrino, which is moving in the $+z$ direction, and the outgoing photon 1 with helicity $\lambda_{1}$. The various helicity-dependent total cross sections are related as

$$
\begin{aligned}
& \sigma_{\lambda \lambda}^{\bar{\nu} \nu \rightarrow \gamma \gamma}=\frac{1}{2} \sigma_{-\lambda-\lambda}^{\gamma \gamma \rightarrow \nu \bar{\nu}} \\
& \sigma_{\lambda-\lambda}^{\bar{\nu} \nu \rightarrow \gamma \gamma}=\sigma_{-\lambda \lambda}^{\gamma \gamma \rightarrow \nu \bar{\nu}}
\end{aligned}
$$

and total cross section for the production of a pair of back-to-back photons can be obtained from

$$
\sigma^{\bar{\nu} \nu \rightarrow \gamma \gamma}=\frac{1}{2 !} \sum_{\lambda_{1} \lambda_{2}} \int_{-1}^{+1} \frac{d \sigma_{\lambda_{1} \lambda_{2}}^{\bar{\nu} \nu \gamma}}{d z} d z .
$$

In view of Eq. (41), we have

$$
\sigma^{\bar{\nu} \nu \rightarrow \gamma \gamma}=2 \sigma^{\gamma \gamma \rightarrow \nu \bar{\nu}} .
$$

\section{DISCUSSION AND CONCLUSIONS}

We have shown that the energy dependence of the cross sections $\sigma^{\nu \gamma \rightarrow \nu \gamma}$ and $\sigma^{\gamma \gamma \rightarrow \nu \bar{\nu}}$ at low energies, $\omega \ll m_{e}$, is the same as that in the energy region, $m_{e} \ll \omega \ll$ $m_{W}$. As a result, the effective interaction introduced in Refs. [8] and [16] contains all the essential of the features of these cross sections. This includes the prediction that the final photons in the channel $\nu \gamma \rightarrow \nu \gamma$ acquire (parity violating) circular polarization.

To investigate the degree of circular polarization of the final photon in the process $\nu \gamma \rightarrow \nu \gamma$, we define the polarization $\mathrm{P}$ as

$$
\mathrm{P}=\frac{\sigma_{--}+\sigma_{+-}-\sigma_{-+}-\sigma_{++}}{\sigma_{--}+\sigma_{+-}+\sigma_{-+}+\sigma_{++}},
$$

where $\sigma_{\lambda_{1} \lambda_{2}} \equiv \sigma_{\lambda_{1} \lambda_{2}}^{\nu \gamma \rightarrow \nu \gamma}$ is defined in Eq. (28). It is clear from Eqs. (29) $-(32)$ that $\sigma_{+-}=\sigma_{-+} \ll \sigma_{--}$, and $\sigma_{--} \simeq$ $2 \sigma_{++}$. Therefore, for photons with the energies $\omega \ll m_{e}$, Eq. (46) gives

$$
\mathrm{P} \simeq \frac{1}{3},
$$

which is independent of the $\omega$. This result in comparable 
about 0.3 for photons with energies $1 \mathrm{GeV} \lesssim \omega \lesssim 10$ $\mathrm{GeV}$.

The angular dependence, $\mathrm{P}(z)$, of the final photon's polarization in the process $\nu \gamma \rightarrow \nu \gamma$, can be obtained from the differential form of Eq. (46),

$$
\mathrm{P}(z)=\frac{d \sigma_{--} / d z-d \sigma_{++} / d z}{d \sigma_{--} / d z+2 d \sigma_{+-} / d z+d \sigma_{++} / d z} .
$$

where the $d \sigma_{\lambda_{1} \lambda_{2}} / d z \equiv d \sigma_{\lambda_{1} \lambda_{2}}^{\nu \gamma \gamma} / d z$ are defined in the Eq. (27), and we have used the equality $d \sigma_{+-} / d z=$ $d \sigma_{-+} / d z$. The polarization $\mathrm{P}(z)$ is plotted in Fig. 6 as a function of $z=\cos \theta$, where $\theta$ is the angle between the incoming and the outgoing neutrinos. In this figure, the solid line is for photons of energy $\omega=m_{e} / 2$, and the dashed line, which is taken from the Ref. [8], is for photons of energy $\omega=10 \mathrm{GeV}$. It is clear from Fig. 6 that the polarization $\mathrm{P}(z)$ remains effectively unchanged for wide range of energies $\omega \lesssim 10 \mathrm{GeV}$. Notice that Eq. (48) can be approximated as

$$
\mathrm{P}(z) \simeq \frac{4-(1+z)^{2}}{4+(1+z)^{2}},
$$

which is independent of the energy of photon.

Our low-energy helicity non-flip amplitudes, obtained from Eqs. (11) and (2) using Eq. (16), enable us to discuss the optical activity of a sea of neutrinos [7]. To do this, it is necessary to establish a relationship between the forward-scattering amplitude and the Lorentz-invariant helicity amplitude for the general case where photons and neutrinos are colliding non-collinearly. Following Møller [17, 18, we define the Lorentz-invariant differential cross section $d \sigma$, for the general non-collinear process $1+2 \rightarrow$ $3+4$ as 19

$$
d \sigma=\frac{|\mathcal{A}(s, t, u)|^{2}}{F} d Q,
$$

where $\mathcal{A}(s, t, u)$ is the Lorentz-invariant amplitude, $F$ is the Lorentz-invariant flux

$$
F=4 \sqrt{\left(p_{1} \cdot p_{2}\right)^{2}-m_{1}^{2} m_{2}^{2}},
$$

and $d Q$ is the Lorentz-invariant phase space differential element

$$
\begin{aligned}
d Q= & (2 \pi)^{4} \delta^{(4)}\left(p_{1}+p_{2}-p_{3}-p_{4}\right) \\
& \times \frac{d^{3} \vec{p}_{3}}{(2 \pi)^{3} 2 E_{3}} \frac{d^{3} \vec{p}_{4}}{(2 \pi)^{3} 2 E_{4}} .
\end{aligned}
$$

After integration over $d^{3} \vec{p}_{4}$, we have

$$
d Q=\frac{\vec{p}_{3}^{2}}{16 \pi^{2} E_{3} E_{4}} \frac{d \Omega_{3}}{\left|\frac{\left|\overrightarrow{p_{3}}\right|}{E_{3}}-\frac{\vec{p}_{3} \cdot \vec{p}_{4}}{\left|\vec{p}_{3}\right| E_{4}}\right|} .
$$

Here, $m_{i}, E_{i}$, and $\vec{p}_{i}$ are the mass, energy, and momentum of the particle $i$ respectively $(i=1.3 .4)$
The resulting $d \sigma$ is essentially identical to Eq. (93) of Ref. [18]. From Eqs. (50), (51), and (53), it is clear that, in the case of forward elastic scattering $\left(m_{3}=\right.$ $m_{1}, m_{4}=m_{2} ; \vec{p}_{3}=\vec{p}_{1}, \vec{p}_{4}=\vec{p}_{2}$ ) and for massless particles $\left(m_{i}=0, i=1,2,3,4\right)$, we have

$$
\left.\frac{d \sigma}{d \Omega_{3}}\right|_{t=0}=\frac{E_{1}^{2}|\mathcal{A}(s, 0,-s)|^{2}}{16 \pi^{2} s^{2}},
$$

where $s=\left(p_{1}+p_{2}\right)^{2}=4 E_{1} E_{2} \sin ^{2}\left(\theta_{12} / 2\right)$, and $\theta_{12}$ is the angle between the momenta $\vec{p}_{1}$ and $\vec{p}_{2}$ of the initial incoming particles 1 and 2. A comparison of Eq. (54) with the cusomary definition of the scattering amplitude, written for forward scattering as

$$
\left.\frac{d \sigma}{d \Omega_{3}}\right|_{t=0}=|f(0)|^{2},
$$

gives

$$
f(0)=\frac{E_{1}}{4 \pi s} \mathcal{A}(s, 0,-s) .
$$

To compute the optical activity of a neutrino sea, we consider a photon of helicity $\lambda$ and energy $\omega$ traversing a bath of neutrinos that are in thermal equilibrium at the temperature $T_{\nu}$. To give an order of magnitude estimate of the index of refraction $n_{\lambda}$ of this sea, we write [20, 21]

$$
n_{\lambda}-1=\frac{2 \pi}{\omega^{2}} \int d N_{\nu} f_{\lambda \lambda}^{\nu \gamma \rightarrow \nu \gamma}(0)
$$

where the forward-scattering amplitude $f_{\lambda \lambda}^{\nu \gamma \rightarrow \nu \gamma}(0)$, from the Eq. (56), is

$$
f_{\lambda \lambda}^{\nu \gamma \rightarrow \nu \gamma}(0)=\frac{\omega}{4 \pi s} \mathcal{A}_{\lambda \lambda}^{\nu \gamma \rightarrow \nu \gamma}(s, 0,-s) .
$$

The Fermi-Dirac distribution, $d N_{\nu}$, is

$$
d N_{\nu}=\frac{1}{(2 \pi)^{3}} \frac{d^{3} \vec{p}_{\nu}}{e^{E_{\nu} / T_{\nu}}+1},
$$

and we have neglected the chemical potential for the neutrinos 22]. Here, $\mathcal{A}_{\lambda \lambda}^{\nu \gamma \rightarrow \nu \gamma}(s, 0,-s)$ is given in the Eqs. (24)-(26), $\vec{p}_{\nu}$ and $E_{\nu}$ are the momentum and energy of a neutrino, and $s=4 \omega E_{\nu} \sin ^{2}\left(\theta_{\nu \gamma} / 2\right)$, where $\theta_{\nu \gamma}$ is the angle between the incoming photon and the incoming neutrino. Therefore, Eqs. (57) and (58) give

$$
n_{\lambda}-1=\int \frac{d N_{\nu}}{2 \omega s} \mathcal{A}_{\lambda \lambda}^{\nu \gamma \rightarrow \nu \gamma}(s, 0,-s) .
$$

After using Eq. (59) for $d N_{\nu}$, Eqs. (24)-(26) for the amplitudes $\mathcal{A}_{\lambda \lambda}^{\nu \gamma \rightarrow \nu \gamma}(s, 0,-s)$, and performing integration in the Eq. (60), we obtain

$$
\begin{aligned}
& n_{+}-1=\frac{T_{\nu}^{4}}{m_{W}^{4}} c_{0}+\frac{\omega T_{\nu}^{5}}{m_{W}^{6}} c_{1}, \\
& n_{-}-1=\frac{T_{\nu}^{4}}{m^{4}} c_{0}-\frac{\omega T_{\nu}^{5}}{m^{6}} c_{1},
\end{aligned}
$$


where (for $\alpha=1 / 137)$

$$
\begin{aligned}
& c_{0}=\frac{7 \alpha^{2} \zeta(4)}{4 \pi^{2} \sin ^{2} \theta_{W}}\left[\ln \left(\frac{m_{W}^{2}}{m_{e}^{2}}\right)+\frac{3}{4}\right] \simeq 1.1 \times 10^{-3},( \\
& c_{1}=\frac{15 \alpha^{2} \zeta(5)}{4 \pi^{2} \sin ^{2} \theta_{W}}\left[\ln \left(\frac{m_{W}^{2}}{m_{e}^{2}}\right)-\frac{8}{3}\right] \simeq 2.0 \times 10^{-3},(64)
\end{aligned}
$$

and $\zeta(x)$ is the Riemann zeta function. The range of the validity of the above relations for the index of refraction, as far as energy is concerned, is related to that of Eqs. (24) $-(26)$, which is $s=4 \omega E_{\nu} \sin ^{2}\left(\theta_{\nu \gamma} / 2\right) \ll m_{W}^{2}$. In Eq. (60), if we change the upper limit of the integration on $E_{\nu}$ from the infinity to $f T_{\nu}$, the contributions of this integral to $c_{0}$ and $c_{1}$ in the Eqs. (61) and (62) change by $9 \%$ and $18 \%$, respectively, if we use $f=7$ (for $f=8$, the corresponding changes are $4 \%$ and 10\%). Here, we set the following criterion

$$
4 \omega f T_{\nu} \ll m_{W}^{2},
$$

which for $f=7$ is

$$
\omega T_{\nu} \ll 2.7 \times 10^{15} \mathrm{GeV} \cdot \mathrm{K},
$$

where $\omega$ is the photon energy in $\mathrm{GeV}$, and $T_{\nu}$ is the neutrino temperature in Kelvin.

From Eqs. 61)-(64), we have

$$
\begin{aligned}
n_{+}-n_{-} & =2 \frac{\omega T_{\nu}^{5}}{m_{W}^{6}} c_{1} \\
& \simeq 7.0 \times 10^{-80} \omega T_{\nu}^{5},
\end{aligned}
$$

and the following approximate relation

$$
\begin{aligned}
n_{+}-1 \simeq n_{-}-1 & \simeq \frac{T_{\nu}^{4}}{m_{W}^{4}} c_{0} \\
& \simeq 1.5 \times 10^{-63} T_{\nu}^{4} .
\end{aligned}
$$

Equation (68) implies that the index of refraction is independent of the helicity and the energy of the incident photon, as long as Eq. (66) is satisfied.

When linearly polarized light propagates through a medium that has different indices of refraction for positive and negative helicities $\left(n_{+} \neq n_{-}\right)$, the plane of polarization of the light rotates by an angle $\phi$, which is [23]

$$
\phi=\frac{\pi}{\lambda_{\gamma}}\left(n_{+}-n_{-}\right) l=\frac{\omega}{2}\left(n_{+}-n_{-}\right) l,
$$

where $\omega$ and $\lambda_{\gamma}=2 \pi / \omega$ are the energy and wavelength of the photon and $l$ is the distance traveled by photons in the medium. To estimate the specific rotary power, $\phi / l$, for a sea of neutrinos, we use Eqs. (67) and (69) to obtain

$$
\begin{aligned}
\frac{\phi}{l} & =\frac{\omega^{2} T_{\nu}^{5}}{m_{W}^{6}} c_{1} \\
& \simeq 1.8 \times 10^{-64} \omega^{2} T_{\nu}^{5} \mathrm{rad} / \mathrm{m} .
\end{aligned}
$$

A positive angle of rotation, $\phi>0$, that is $n_{+}>n_{-}$, corresponds to a clockwise rotation (dextrorotation) of the plane of polarization of the linearly polarized incident photons, as viewed by an observer that is detecting the forward-scattered light. Thus, the optical activity of a neutrino sea is that of a dextrorotary medium. In addition, it is clear from Eq. (70) that the rotary power, $\phi / l$, varies as $1 / \lambda_{\gamma}^{2}$, which is the same as that of quartz and most transparent substances for visible light.

To get a rough estimate of rotation angle $\phi$ for linearly polarized photons propagating through the relic neutrino sea, we use Eq. (70) with $l=c t, c=3 \times 10^{8} \mathrm{~m} / \mathrm{s}, t \sim$ $15 \times 10^{9}$ years, $T_{\nu} \sim 2 \mathrm{~K}$, and $\omega \sim 10^{20} \mathrm{eV}$, and for the neutrino part of the sea we find

$$
\phi \sim 8 \times 10^{-15} \mathrm{rad},
$$

which is exceedingly small. The antineutrino part of the sea gives a rotation with opposite sign, such that if the asymmetry parameter [24], $L \equiv\left(N_{\nu}-N_{\bar{\nu}}\right) / N_{\gamma}$, is zero, the resultant angle of rotation $\phi$ will be zero. The proper treatment for the case $L \neq 0$, is to include the chemical potential in the neutrino and antineutrino distribution functions.

\section{Acknowledgments}

We wish to thank Duane Dicus for numerous helpful discussions. One of us (A.A.) wishes to thank the Department of Physics and Astronomy at Michigan State University for its hospitality and computer resources. This work was supported in part by the National Science Foundation under Grant No. PHY-0070443.
[1] H.-Y. Chiu and P. Morrison, Phys. Rev. Lett. 5, 573 (1960).

[2] M. J. Levine, Nuovo Cimento 48A, 67 (1967).

[3] L. F. Landovitz and W. M. Schreiber, Nuovo Cimento 2A, 359 (1971).

[4] V. K. Cung and M. Yoshimura, Nuovo Cimento 29A, $557(1975)$
[5] D. A. Dicus and W. W. Repko, Phys. Rev. D 48, 5106 (1993).

[6] J. Liu, Phys. Rev. D 44, 2879 (1991).

[7] G. Karl and V. Novikov, "Optical activity of a neutrino sea in the Standard Model," hep-ph/0009012.

[8] A. Abbasabadi, A. Devoto, and W. W. Repko, Phys. Rev. 
[9] For a related decomposition in the case $\nu^{\prime} \rightarrow \nu \gamma \gamma$, see $\mathrm{J}$. F. Nieves, Phys. Rev. D 28, 1664 (1983).

[10] A. Abbasabadi, A. Devoto, D. A. Dicus, and W. W. Repko, Phys. Rev. D 59, 013012 (1999).

[11] M. B. Gavela, G. Girardi, C. Malleville, and P. Sorba, Nucl. Phys. B193, 257 (1981); M. Bace and N. D. Hari Dass, Ann. of Phys. 94, 349 (1975).

[12] J. F. Nieves, P. B. Pal, and D. G. Unger, Phys. Rev. D 28, 908 (1983).

[13] J. A. M. Vermaseren, "New features of FORM," math$\mathrm{ph} / 0010025$.

[14] M. J. G. Veltman, "SCHOOnschip A Program for Symbol Handling," University of Michigan, report, 1984 (unpublished). See also, M. Veltman and D. N. Williams, "Schoonschip '91," hep-ph/9306228.

[15] D. Seckel, Phys. Rev. Lett. 80, 900 (1998).

[16] D. A. Dicus, K. Kovner and W. W. Repko, Phys. Rev. D 62, 053013 (2000).

[17] C. Møller, Danske Videnskab. Selskab 23, No. 1 (1945).

[18] For a reprint of the Ref.[17], see M. H. Ross, Quantum Scattering Theory (Indiana University Press, Bloomington, Ind. 1963), page 109.

[19] For a brief disscusion of differential cross section for noncollinear scattering, see R. G. Newton, Scattering Theory of Waves and Particles (McGraw-Hill, NY, 1966), page 220. See also page 91 of the Ref. [21].

[20] Notice that Eq. (57) is based on the standard Lorentz relation that connects the index of refraction to the forward-scattering amplitude, for a medium that is at $T=0$ temperature. Here, we make a nontrivial assumption that the introduction of finite temperature in the medium, will not spoil the coherent condition that was necessary to derive the Lorentz relation. For a similar approach, see P. Langacker and J. Liu, Phys. Rev. D 46, 4140 (1992).

[21] M. L. Goldberger and K. M. Watson, Collision Theory (Wiley, NY, 1964), page 771.

[22] P. J. E. Peebles, Principles of Physical Cosmology (Princeton University Press, Princeton, NJ, 1993), page 160.

[23] For a discussion of the rotation of the polarization plane of a linearly polarized light in a gas, see the discussion leading to the Eq. (8) of V. G. Baryshevsky, "Timereversal-violating birefringence of photon in a medium exposed to electric and magnetic field", hep-ph/0007353. For a general discussion of rotary power, see J. P. Mathieu, Optics (Pergamon Press, NY, 1975), page 235; M. V. Klein, Optics (John Wiley \& Sons, NY, 1970), page 502.

[24] J. Lesgourgues and S. Pastor, Phys. Rev. D 60, 103521 (1999).

\section{Figures}



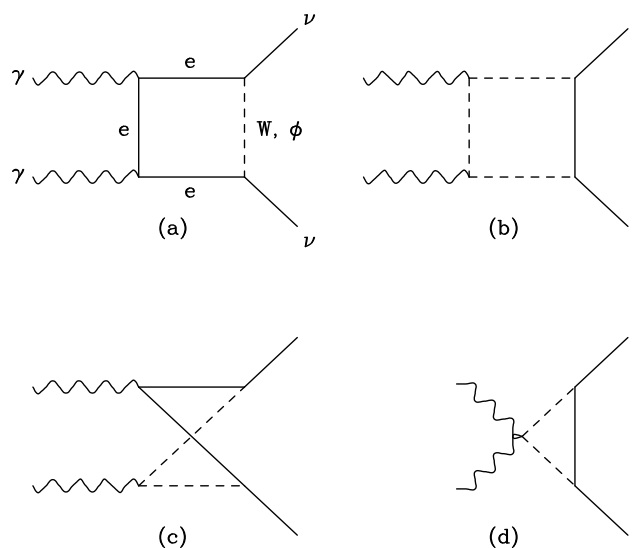

FIG. 1: Diagrams for $\nu_{e} \gamma \rightarrow \nu_{e} \gamma$ or $\gamma \gamma \rightarrow \nu_{e} \bar{\nu}_{e}$. The diagram (d) will give zero contribution. For each of (a), (b), (c) there is also a diagram with the photons interchanged.

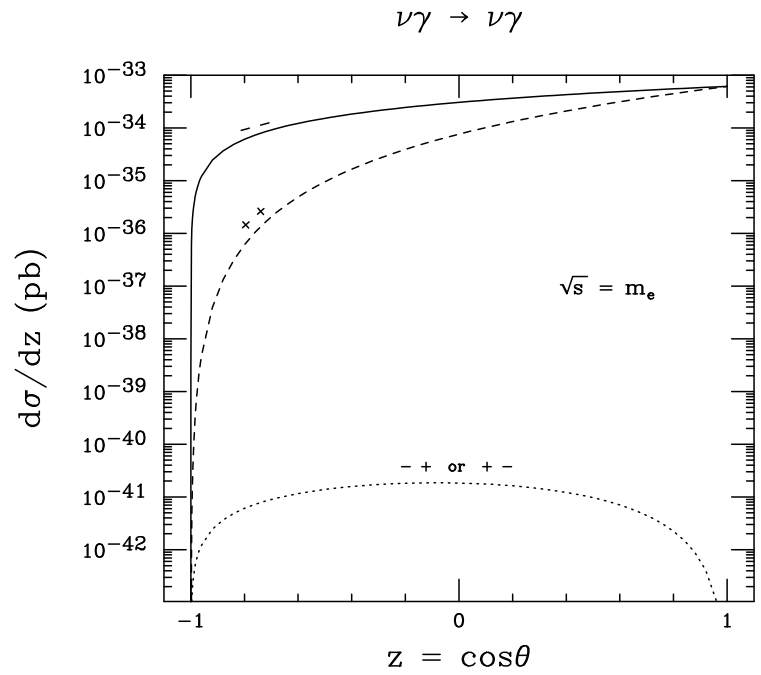

FIG. 2: The helicity dependent differential cross sections for $\nu \gamma \rightarrow \nu \gamma$ are shown for $\sqrt{s}=m_{e}$. The solid line is $d \sigma_{--} / d z$, the dashed line is $d \sigma_{++} / d z$, and the dotted line is $d \sigma_{+-} / d z$. The $d \sigma_{-+} / d z$ is the same as $d \sigma_{+-} / d z$. 


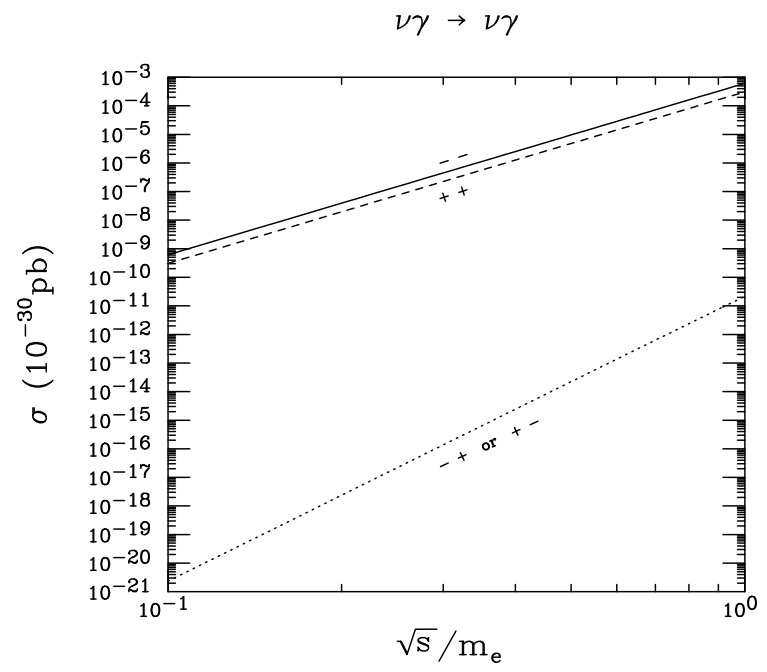

FIG. 3: The helicity dependent total cross sections for $\nu \gamma \rightarrow$ $\nu \gamma$ are shown. The solid line is $\sigma_{--}$, the dashed line is $\sigma_{++}$, and the dotted line is $\sigma_{+-}$. The $\sigma_{-+}$is the same as $\sigma_{+-}$.

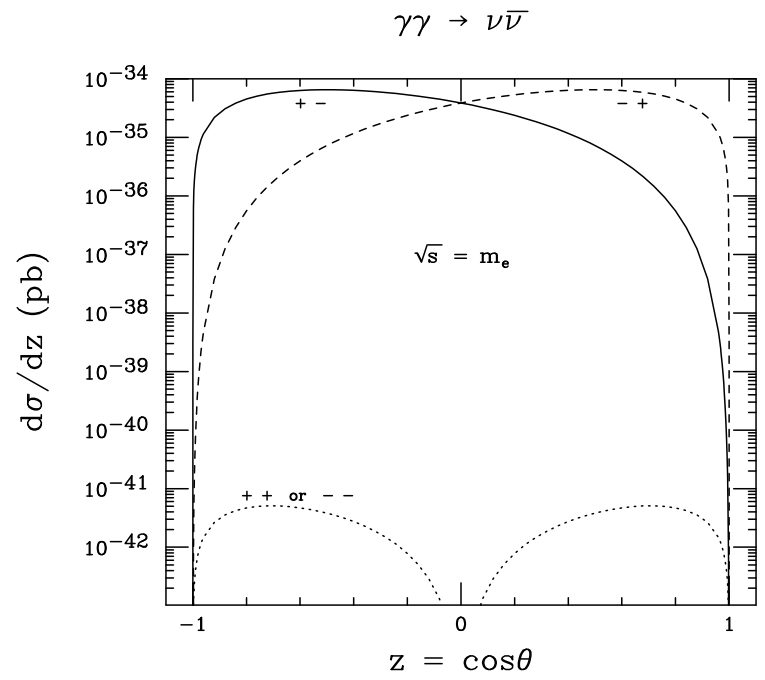

FIG. 4: The helicity dependent differential cross sections for $\gamma \gamma \rightarrow \nu \bar{\nu}$ are shown for $\sqrt{s}=m_{e}$. The solid line is $d \sigma_{+-} / d z$, the dashed line is $d \sigma_{-+} / d z$, and the dotted line is $d \sigma_{++} / d z$.

The $d \sigma_{--} / d z$ is the same as $d \sigma_{++} / d z$. 


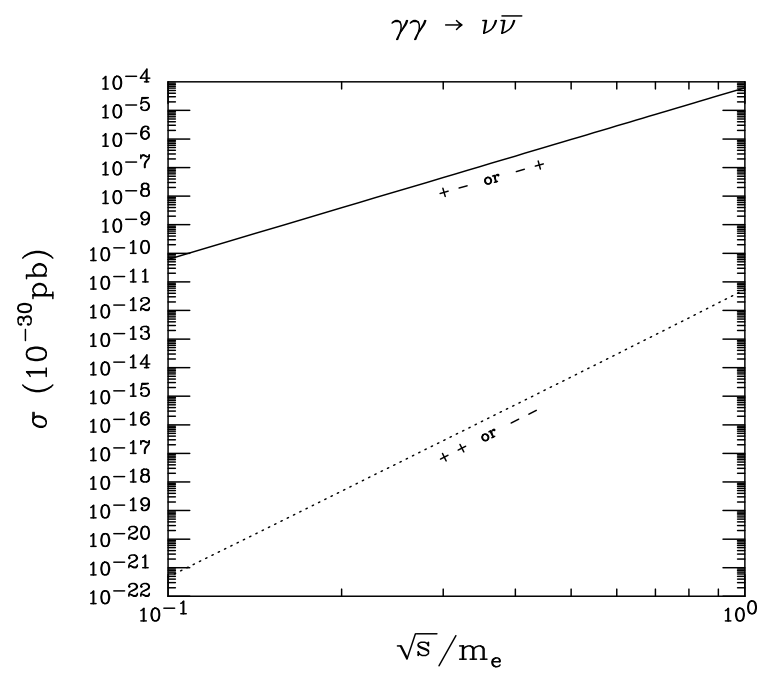

FIG. 5: The helicity dependent total cross sections for $\gamma \gamma \rightarrow$ $\nu \bar{\nu}$ are shown. The solid line is $\sigma_{+-}$and the dotted line is $\sigma_{++}$. The $\sigma_{-+}$is the same as $\sigma_{+-}$, and $\sigma_{--}$is the same as $\sigma_{++}$.

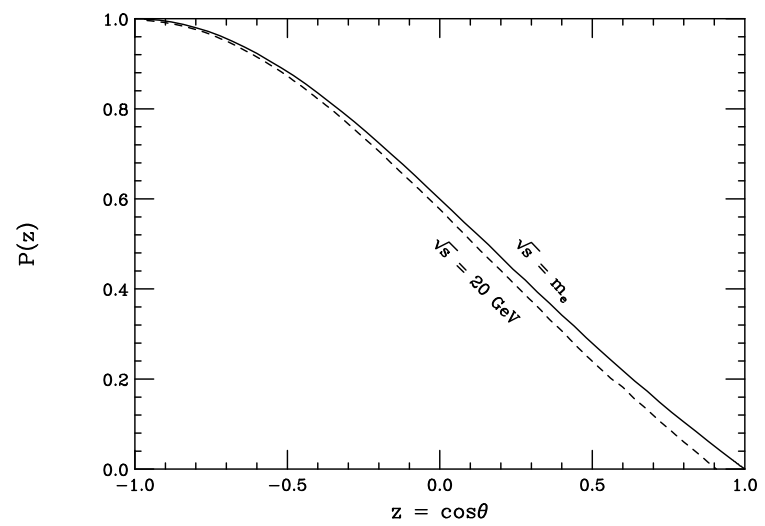

FIG. 6: The polarization $\mathrm{P}(\mathrm{z})$ of the final photons in the process $\nu \gamma \rightarrow \nu \gamma$, as it is defined in the Eq. 48 ), is shown. The solid line is polarization for the center of mass energy $\sqrt{s}=m_{e}$, while the dashed line is for the center of mass energy $\sqrt{s}=20 \mathrm{GeV}$, which is taken from the Ref. [8]. 